\title{
Colorectal cancer, comorbidity, and risk of venous thromboembolism: assessment of biological interactions in a Danish nationwide cohort
}

\author{
Thomas P Ahern*,1, Erzsébet Horváth-Puhó ${ }^{2}$, Karen-Lise Garm Spindler ${ }^{3}$, Henrik Toft Sørensen ${ }^{2}$, \\ Anne G Ording ${ }^{2}$ and Rune Erichsen ${ }^{2}$ \\ ${ }^{1}$ Departments of Surgery and Biochemistry, University of Vermont College of Medicine, 89 Beaumont Avenue, Given D317A, \\ Burlington, VT 05405, USA; ${ }^{2}$ Department of Clinical Epidemiology, Aarhus University Hospital, Olof Palmes Allé 43-45, DK-8200 \\ Aarhus N, Denmark and ${ }^{3}$ Department of Oncology, Aarhus University Hospital, Nørrebrogade 44, DK-8000 Aarhus C, Denmark
}

Background: Venous thromboembolism (VTE) is a major source of morbidity and mortality in cancer patients. Incident colorectal cancer (CRC) and comorbidity both predict VTE, but potential synergy between these factors has not been explored.

Methods: Danish nationwide cohort study of CRC cases diagnosed in 1995-2010 and a matched general population reference cohort of subjects without CRC who matched cases on age, sex, and comorbidities. We calculated the Charlson Comorbidity Index using diagnoses recorded in the Danish National Patient Registry. We calculated standardised incidence rates (SIRs) and interaction contrasts $(\mathrm{IC})$ to measure additive interaction between comorbidity and CRC status with respect to 5-year VTE incidence.

Results: Among 56189 CRC patients, 1372 VTE cases were diagnosed over 145211 person-years (SIR=9.5 cases per 1000 personyears). Among 271670 reference subjects, 2867 VTE cases were diagnosed over 1068860 person-years (SIR =2.8 cases per 1000 person-years). CRC and comorbidity were positively and independently associated with VTE, but there was no evidence for biological interaction between these factors (e.g., comparing the 'severe comorbidity' stratum with the 'no comorbidity' stratum, $\mathrm{IC}=0.8,95 \% \mathrm{Cl}:-3.3,4.8)$.

Conclusions: There is neither a deficit nor a surplus of VTE cases among patients with both comorbidity and CRC, compared with rates expected from these risk factors in isolation.

Venous thromboembolism (VTE) affects about one per thousand adults each year (Cushman, 2007). Risk increases markedly with age and is higher for men than for women (Cushman, 2007). In the worst-case scenario, VTE results in death; and even in the best-case scenario, VTE can cause considerable morbidity and increase the risk of death several decades after diagnosis (Kahn et al, 2002; Cushman, 2007; Søgaard et al, 2014). The pro-thrombotic state associated with malignancy leaves cancer survivors at much higher
VTE risk than similar cancer-free individuals (Gouin-Thibault et al, 2001; Blom et al, 2005; Prandoni et al, 2005). The degree of elevated VTE risk depends on anatomical site, stage, and grade of malignancy (Alcalay et al, 2006; Chew et al, 2006; Khorana et al, 2007; Lemmens et al, 2007; Wun and White, 2009; Kyriazi and Theodoulou, 2013; Konigsbrugge et al, 2014). Prevalent comorbidities among cancer survivors further increase VTE risk-either directly or as a consequence of hospitalisation, surgery, and/or

*Correspondence: Dr TP Ahern; E-mail: thomas.ahern@med.uvm.edu

Received 6 July 2015; revised 14 October 2015; accepted 2 November 2015; published online 1 December 2015

(c) 2016 Cancer Research UK. All rights reserved 0007 - 0920/16 
immobility (Alcalay et al, 2006; Khorana et al, 2007; Lemmens et al, 2007; Wun and White, 2009; Khorana and Connolly, 2009; Kyriazi and Theodoulou, 2013; Konigsbrugge et al, 2014). For example, in a cohort of hospitalised cancer patients, pulmonary disease and active infection increased the odds of VTE by $37 \%$ and $77 \%$, respectively (Khorana et al, 2007). Improving primary prevention of VTE in CRC survivors requires identification of high-risk individuals, for whom the potential benefits of anticoagulation therapy outweigh the potential risks (Ay et al, 2010; Kyriazi and Theodoulou, 2013). Although earlier studies have examined VTE risk as a function of comorbidity among cancer patients, none has evaluated the impact of interaction between a diagnosis of colorectal cancer (CRC) and prevalent comorbidity on VTE occurrence. Such interaction would have strong implications for clinical assessment and VTE primary prevention among CRC patients, among whom comorbidity is quite common.

We conducted a Danish nationwide cohort study to assess the effect of additive interaction between CRC and comorbidity on VTE incidence. Specifically, we evaluated whether the VTE rate among CRC patients with prevalent comorbidity differed from the sum of the VTE rate in CRC patients without comorbidity and the VTE rate in adults without CRC but with prevalent comorbidity.

\section{MATERIALS AND METHODS}

This study was approved by the Danish Data Protection Agency (journal number 2011-41-5809).

Source population and data collection. We conducted a nationwide, population-based, matched cohort study using Danish medical and civil registries to assess the impact of biological interaction between CRC and comorbidity on VTE occurrence. The Danish National Health Service provides tax-supported healthcare to the country's entire population $(\sim 5.6$ million people). Within this healthcare system, specialised registries track diagnoses, procedures, prescriptions, and vital status throughout the population. Individual-level data from these registries can be linked unambiguously using the unique civil personal registration (CPR) number assigned to all Danish residents (Frank, 2000).

We used the Danish Cancer Registry (DCR), which has recorded cancer diagnoses since 1943, to enumerate a cohort of all the CRC cases diagnosed between 1995 and 2010 (Storm et al, 1997). We linked this cohort to the Danish National Patient Registry (DNPR; Andersen et al, 1999) - which has recorded diagnoses made during non-psychiatric hospital admissions since 1977 and during hospital outpatient and emergency visits since 1995 - to exclude CRC cases with a prior or coincident VTE diagnosis $(n=1798)$. We used the DCR to ascertain each subject's date of diagnosis, cancer stage, and anatomical site of disease.

We linked the DCR to the DNPR and the Danish Civil Registration System (DCRS; Pedersen et al, 2006; Schmidt et al, 2014), which tracks vital status and residence, to match up to five reference subjects to each CRC patient. The index date for CRC patients was their date of cancer diagnosis. Reference subjects were sampled at random from the set of individuals who had no history of CRC or VTE on the CRC patient's index date, and who matched the CRC patient on age ( \pm 5 years), sex, and single comorbidities (Table 1). Reference subjects were assigned the same index date as their matched CRC patient. CRC patients with no eligible matched reference subject were excluded.

Person-time at risk was counted from the index date until the first of the following events: inpatient VTE diagnosis, emigration from Denmark, death, five years of follow-up, or the end of the study period (31 December 2011). Subjects in the reference cohort who were diagnosed with CRC during follow-up became part of the CRC cohort, provided they had not been diagnosed with VTE.

Table 1. Characteristics of colorectal cancer cases and the matched reference cohort, Denmark, 1995-2010

\begin{tabular}{|c|c|c|}
\hline Characteristics & $\begin{array}{c}\text { Colorectal } \\
\text { cancer cases } \\
\begin{array}{c}N=56189 \\
n(\%)\end{array}\end{array}$ & $\begin{array}{c}\text { Matched } \\
\text { cohort } \\
N=271670 \\
n(\%)\end{array}$ \\
\hline \multicolumn{3}{|l|}{ Sex } \\
\hline Female & $27350(49)$ & $132537(49)$ \\
\hline Male & 28839 (51) & $139133(51)$ \\
\hline \multicolumn{3}{|c|}{ Age on index date (years) } \\
\hline$\leqslant 49$ & $2805(5.0)$ & $14316(5.3)$ \\
\hline $50-59$ & 7426 (13) & $37151(14)$ \\
\hline $60-69$ & $14407(26)$ & $70613(26)$ \\
\hline 70-79 & $18276(33)$ & 87444 (32) \\
\hline$\geqslant 80$ & $13275(24)$ & $62146(23)$ \\
\hline
\end{tabular}

\section{Stage of cancer}

Non-metastatic

Metastatic

(missing)

$36963(75)$

$12503(25)$

6723

\section{Cancer site}

\section{Colon cancer}

Rectal cancer

Colon and rectal cancer

(coincident)

\section{Index date}

37275 (66)

$18826(34)$

$88(0.2)$

NA

NA

1995-1999

2000-2004

2005-2010

\section{Prevalent comorbidities}

\section{Atrial fibrillation}

Congestive heart failure

Myocardial infarction

Peripheral vascular disease

Cerebrovascular disease

Chronic pulmonary disease

Connective tissue disease

Ulcer disease

Mild liver disease

Moderate/severe liver disease

Diabetes without complications

Diabetes with end-organ damage

Moderate/severe renal disease

Other solid tumour, invasive

Other solid tumour, metastatic

Leukaemia

Lymphoma

AIDS

Dementia

Hemiplegia

Obesity, clinically diagnosed

Charlson Comorbidity Index

\section{No comorbidity}

Mild comorbidity

Moderate comorbidity

Severe comorbidity

Abbreviation: $\mathrm{NA}=$ not available.

16061 (29)

17152 (31)

22976 (41)

$985(1.8)$

2474 (4.4)

3050 (5.4)

2111 (3.8)

4745 (8.4)

3755 (6.7)

1408 (2.5)

2798 (5.0)

$378(0.7)$

$79(0.1)$

2691 (4.8)

1164 (2.1)

$636(1.1)$

4805 (8.6)

$434(0.8)$

$116(0.2)$

$236(0.4)$

$7(0.01)$

$518(0.9)$

$65(0.1)$

1007 (1.8)

$78136(29)$

83088 (31)

110446 (41)

Definition of analytic variables. International Classification of Disease (ICD) codes were used to identify cases of CRC, VTE, and specific comorbidities (codes are available from the corresponding author). The CRC cases were further characterised by anatomical site of disease (colon or rectum). Stage at diagnosis was defined as metastatic or non-metastatic. Age on the index date was categorised for use in stratified analyses $(\leqslant 49,50-59,60-69$, $70-79$, and $\geqslant 80$ years), but was modelled as a continuous variable in regression-based analyses. Venous thromboembolism was a composite end point comprising incident pulmonary embolism (PE) or deep vein thrombosis (DVT). Colorectal resection was 
identified using the following surgical codes in the DNPR: 4482045081, 45840-45900, KJFB 20-97, KJFH, and KJGB.

Individual comorbidities were defined as positive for study subjects if any corresponding ICD code was recorded in the DNPR between 1977 and a subject's index date. We also summarised comorbidity burden using a modified version of the Charlson Comorbidity Index (CCI; Charlson et al, 1987). The original CCI algorithm is based on 19 diagnostic classes comprising cardiovascular, metabolic, hepatic, and renal diseases, as well as malignancies, dementia, stomach ulcers, and AIDS. Our modified CCI included two new diagnoses - atrial fibrillation and obesity - and excluded colorectal cancer from the set of malignancies. Each prevalent comorbidity was assigned a severity weight according to the CCI algorithm (we assigned weights of 1 to both atrial fibrillation and obesity). The sum of an individual's severity weights was then translated into ordinal CCI categories representing no comorbidity (sum of weights equal to 0 ), mild comorbidity (sum of weights equal to 1 ), moderate comorbidity (sum of weights equal to 2 or 3 ), and severe comorbidity (sum of weights greater than 3 ).

Statistical analysis. We tabulated the frequency and proportion of CRC cases and reference subjects according to age group, sex, index date range, and comorbidity (Table 1). We calculated 5-year standardised VTE incidence rates (SIRs) overall and within CCI strata. Rates were standardised to the age and sex distribution of the CRC cohort.

We fit Cox proportional hazards models adjusted for age, sex, and calendar year of CRC diagnosis to estimate associations between CRC diagnosis and VTE incidence, both overall (adjusting for CCI) and within CCI strata. In these models, subjects were censored if they died, emigrated from Denmark, contributed 5 years of follow-up, or reached the end of the study period without a VTE event. We report associations as hazard ratios (HRs) with corresponding Wald 95\% confidence intervals (95\% CIs). We verified the proportionality of hazard functions by visually evaluating plots of log-negative log survival as a function of $\log$ follow-up time.

We also calculated interaction contrasts (ICs) to assess the biological interaction between a CRC diagnosis and prevalent comorbidity on VTE risk (Greenland et al, 2008). The IC disaggregates the VTE incidence rate into three components: (1) the rate increase due to CRC only, (2) the rate increase due to comorbidity only, and (3) the background VTE rate among subjects with neither CRC nor comorbidity. If the rate among the doubly exposed (subjects with both CRC and comorbidity) is equal to the sum of the three components $(\mathrm{IC}=0)$, there is no biological interaction between CRC and comorbidity. However, if the rate among doubly exposed patients is greater than the sum of the three components (IC>0), CRC and comorbidity act synergistically to increase the rate of VTE beyond the sum of their individual effects. Finally, if the rate among the doubly unexposed is less than the sum of the three components $(\mathrm{IC}<0), \mathrm{CRC}$ and comorbidity counteract one another, reducing the rate of VTE compared with the sum of their individual effects.

We calculated ICs based on CCI-stratified SIRs, overall and within strata of VTE type (PE, DVT, and other types), stage at diagnosis (metastatic or non-metastatic), and anatomical site of cancer (colon or rectum). As the CCI score is a four-level variable, separate ICs were calculated for mild, moderate, and severe comorbidity, each using 'no comorbidity' as the reference. Variances for the IC estimates equaled the summed variances of the component SIRs (Greenland et al, 2008). As an example, consider the IC of -0.2 reported for CRC and mild comorbidity (Table 2). This value is calculated by taking the VTE SIR in the doubly exposed $(\mathrm{CRC}+$ mild comorbidity; SIR $=9.8$ cases per 1000 person-years (PY)) and subtracting from it (1) the rate increase owing to $\mathrm{CRC}$ (the $\mathrm{CRC} /$ reference rate difference in the 'no comorbidity' stratum: difference $=9.0-2.3=6.7$ cases per $1000 \mathrm{PY}$ ), (2) the rate increase owing to comorbidity (the mild comorbidity/no comorbidity rate difference among reference subjects: difference $=3.3-2.3=1.0$ case per $1000 \mathrm{PY}$ ), and (3) the baseline VTE rate in reference subjects with no comorbidity (2.3 cases per $1000 \mathrm{PY}$ ).

To address the heightened risk of VTE after surgery, we evaluated interactions between CRC and comorbidity using the subset of CRC patients who underwent colorectal resection within 90 days of their diagnosis date and their matched reference subjects. For this analysis, we divided follow-up into two risk periods: $\leqslant 90$ days after resection and $>90$ days after resection (up to a maximum of 5 years). We restricted the analysis to patients undergoing CRC resection because there is evidence that VTE risk is modified by type of abdominal surgery (Mukherjee et al, 2008). We conducted a sensitivity analysis in which VTE cases were defined as those diagnosed in both the hospital inpatient and outpatient settings. Finally, since thromboprophylaxis protocols have varied over time, we repeated the analyses within the strata of calendar period (1995-2002 and 2003-2010).

Table 2. Five-year rates of incident venous thromboembolism by colorectal cancer status, overall and according to comorbidity level, Denmark, 1995-2010

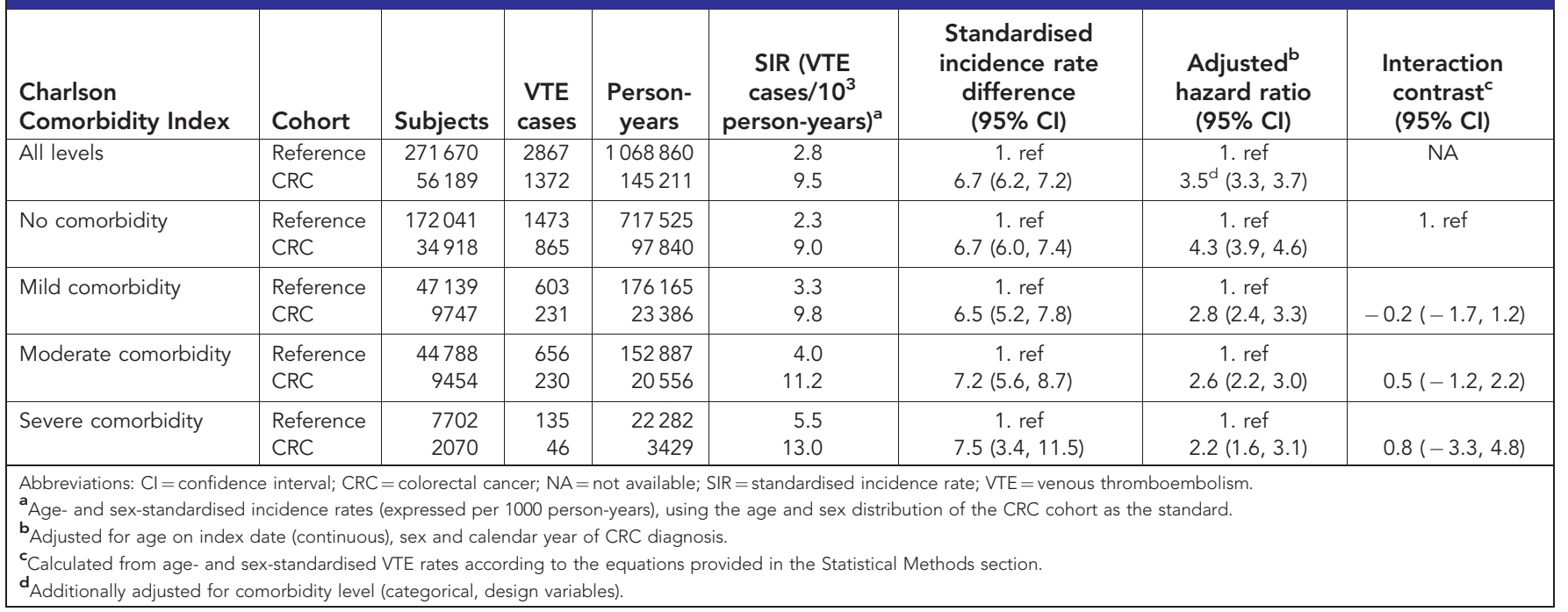


The analyses were carried out using SAS software, version 9.2 (SAS Institute, Cary, NC, USA).

\section{RESULTS}

We identified 56963 CRC patients diagnosed during 1995-2010. Of these, $774(1.4 \%)$ were excluded owing to lack of suitable matched reference subjects. The final cohort included 56189 CRC patients, to whom 271670 reference subjects were matched. Each CRC patient had at least one matched reference subject (range $=1$ to 5 , mode $=5$ ).

Table 1 shows the distribution of the CRC and reference cohorts according to key analytic variables. The CRC and reference cohorts had similar or identical distributions of sex, age, index year, and prevalent comorbidities - all as expected owing to the matching (subtle differences in proportions arise from the unequal matching ratio).

Colorectal cancer, comorbidity, and 5-year incidence of venous thromboembolism. Associations between CRC, comorbidity, and 5-year VTE incidence are shown in Table 2. The CRC patients contributed 145211 PY of observation (median $=2.3$ years), during which 1372 cases of VTE were diagnosed (SIR $=9.5$ cases per $1000 \mathrm{PY})$. Reference subjects contributed $1068860 \mathrm{PY}$ of observation (median $=5.0$ years), during which 2867 cases of VTE were diagnosed ( $\mathrm{SIR}=2.8$ cases per $1000 \mathrm{PY}$ ). Without stratification on comorbidity, CRC was associated with a 3.5 -fold increased rate of incident VTE (adjusted $\mathrm{HR}=3.5,95 \% \mathrm{CI}$ : 3.3, 3.7), compared with the reference cohort. VTE incidence rates among CRC patients increased only slightly across strata of comorbidity, ranging from 9.0 events per 1000 PY in the 'no comorbidity' stratum to 13.0 events per $1000 \mathrm{PY}$ in the 'severe comorbidity' stratum.

Comorbidity was positively and independently associated with VTE. Within the reference cohort, the standardised VTE incidence rate increased monotonically with comorbidity level (e.g., in the 'no comorbidity' stratum, SIR $=2.3$ cases per $1000 \mathrm{PY}$ and in the 'severe comorbidity' stratum, SIR $=5.5$ cases per $1000 \mathrm{PY}$ ). As a consequence of these patterns, HRs associating CRC diagnosis with VTE became weaker in the strata of increasing comorbidity burden.

We saw no evidence of biological interaction between CRC and comorbidity level with respect to VTE incidence (for mild comorbidity, $\mathrm{IC}=-0.2$, 95\% CI: $-1.7,1.2$; for moderate comorbidity, IC $=0.5,95 \% \mathrm{CI}:-1.2,2.2$; and for severe comorbidity, IC $=0.8,95 \% \mathrm{CI}:-3.3,4.8)$. These ICs are consistent with neither a deficit nor a surplus of VTE cases among individuals exposed to both CRC and at least some comorbidity.

Supplementary Table 1 and Supplementary Figure 1 show interaction contrast estimates for specific prevalent comorbidities. As these estimates do not exclude subjects with comorbidities other than those of specific interest (owing to sample size limitations), these results should be interpreted with caution.

Post-resection risk period analysis. Table 3 shows associations during earlier and later follow-up periods after CRC resection. This analysis was restricted to CRC patients who underwent colorectal resection within 90 days of their diagnosis $(n=25261)$ and their matched reference subjects $(n=124340)$.

In the first 90 days of post-resection follow-up, the standardised VTE rate among CRC patients with no comorbidity was 26.0 events per $1000 \mathrm{PY}$. The rate was similar among CRC patients with severe comorbidity (24.8 cases per 1000 PY). Among reference subjects, the standardised VTE rate in the 'no comorbidity' stratum was 1.8 events per $1000 \mathrm{PY}$ and rates increased with comorbidity level (e.g., in the 'severe comorbidity' stratum, SIR $=3.8$ VTE cases per $1000 \mathrm{PY})$.

In the later follow-up period (91 days to 5 years post resection), the comorbidity stratum-specific VTE rates among reference subjects were similar to the corresponding rates in the early follow-up period. However, VTE rates were considerably lower for

Table 3. Associations between colorectal cancer diagnosis and venous thromboembolism stratified by Charlson Comorbidity Index levels, according to earlier ( $\leqslant 90$ days) and later (between 91 days and 5 years) risk periods following colorectal resection, Denmark, 1995-2010

\begin{tabular}{|c|c|c|c|c|c|c|c|c|}
\hline $\begin{array}{l}\text { Comorbidity } \\
\text { stratum }\end{array}$ & Cohort & Subjects & $\begin{array}{l}\text { VTE } \\
\text { cases }\end{array}$ & $\begin{array}{l}\text { Person- } \\
\text { years }\end{array}$ & $\begin{array}{l}\text { SIR }(\mathrm{VTE} \\
\text { cases } / 10^{3} \\
\text { person-years) }\end{array}$ & $\begin{array}{l}\text { Standardised } \\
\text { incidence rate } \\
\text { difference } \\
(95 \% \mathrm{Cl})\end{array}$ & $\begin{array}{l}\text { Adjusted }^{b} \\
\text { hazard ratio } \\
(95 \% \mathrm{Cl})\end{array}$ & $\begin{array}{l}\text { Interaction } \\
\text { contrast } \\
(95 \% \mathrm{Cl})\end{array}$ \\
\hline No comorbidity & $\begin{array}{l}\text { Reference } \\
\text { CRC }\end{array}$ & $\begin{array}{r}124340 \\
25261\end{array}$ & $\begin{array}{r}48 \\
148\end{array}$ & $\begin{array}{r}30556 \\
5857\end{array}$ & $\begin{array}{r}1.8 \\
26.0\end{array}$ & $\begin{array}{c}\text { 1. ref } \\
24.2(19.9,28.5)\end{array}$ & $\begin{array}{c}\text { 1. ref } \\
16(12,22)\end{array}$ & 1. ref \\
\hline Mild comorbidity & $\begin{array}{l}\text { Reference } \\
\text { CRC }\end{array}$ & $\begin{array}{r}32737 \\
6787\end{array}$ & $\begin{array}{l}20 \\
41\end{array}$ & $\begin{array}{l}8016 \\
1494\end{array}$ & $\begin{array}{r}2.2 \\
27.4\end{array}$ & $\begin{array}{c}\text { 1. ref } \\
25.2(16.5,33.9)\end{array}$ & $\begin{array}{c}\text { 1. ref } \\
11(6.4,19)\end{array}$ & $1.0(-8.7,11)$ \\
\hline Severe comorbidity & $\begin{array}{l}\text { Reference } \\
\text { CRC }\end{array}$ & $\begin{array}{l}4662 \\
1265\end{array}$ & $\begin{array}{l}5 \\
8\end{array}$ & $\begin{array}{r}1121 \\
263\end{array}$ & $\begin{array}{r}3.8 \\
24.8\end{array}$ & $\begin{array}{c}\text { 1. ref } \\
21.0(2.3,39.7)\end{array}$ & $\begin{array}{c}\text { 1. ref } \\
7.0(2.3,21)\end{array}$ & $-3.2(-22,16)$ \\
\hline \multicolumn{9}{|c|}{$>90$ days after CRC resection } \\
\hline No comorbidity & $\begin{array}{l}\text { Reference } \\
\text { CRC }\end{array}$ & $\begin{array}{r}123644 \\
23108\end{array}$ & $\begin{array}{l}966 \\
416\end{array}$ & $\begin{array}{r}490256 \\
73727\end{array}$ & $\begin{array}{l}2.2 \\
5.7\end{array}$ & $\begin{array}{c}\text { 1. ref } \\
3.5(2.9,4.1)\end{array}$ & $\begin{array}{c}\text { 1. ref } \\
2.9(2.6,3.2)\end{array}$ & 1. ref \\
\hline Severe comorbidity & $\begin{array}{l}\text { Reference } \\
\text { CRC }\end{array}$ & $\begin{array}{r}4436 \\
996\end{array}$ & $\begin{array}{l}91 \\
23\end{array}$ & $\begin{array}{r}12465 \\
2350\end{array}$ & $\begin{array}{r}6.7 \\
10.1\end{array}$ & $\begin{array}{c}\text { 1. ref } \\
3.4(-1.2,8.0)\end{array}$ & $\begin{array}{c}\text { 1. ref } \\
1.4(0.85,2.1)\end{array}$ & $-0.1(-4.7,4.5)$ \\
\hline \multicolumn{9}{|c|}{ 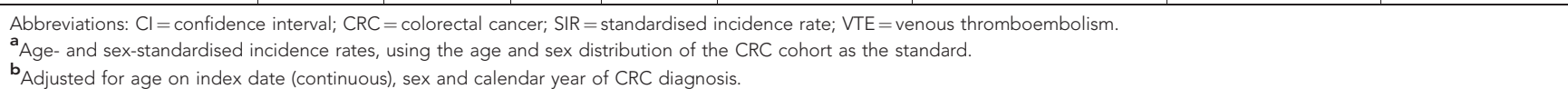 } \\
\hline
\end{tabular}


CRC patients during the later period, ranging from 5.7 cases per $1000 \mathrm{PY}$ in the 'no comorbidity' stratum to 10.1 cases per $1000 \mathrm{PY}$ in the 'severe comorbidity' stratum (Table 3).

There was no suggestion of biological interaction between CRC and comorbidity in either the earlier or the later follow-up periods.

Modification by type of venous thromboembolism, stage at diagnosis, cancer site, and time since surgery. Of the 4239 VTE events diagnosed during follow-up, 2064 (49\%) were cases of PE and 2175 (51\%) were cases of DVT. Figure 1 shows ICs between CRC and comorbidity according to these different event types. For $\mathrm{PE}$, there was a positive trend in the IC across increasing levels of comorbidity, indicating that CRC and comorbidity act synergistically to generate more cases of PE than would be expected from the two exposures acting independently. For DVT, there was a negative trend in IC across increasing levels of comorbidity, indicating that $\mathrm{CRC}$ and comorbidity counteract one another, resulting in fewer cases of DVT than would be expected from the two exposures acting independently.

Among the 49466 members of the CRC cohort with known cancer stage, 36963 (75\%) were diagnosed with non-metastatic disease and 12503 (25\%) were diagnosed with metastatic disease. Figure 2 depicts ICs between comorbidity and either non-

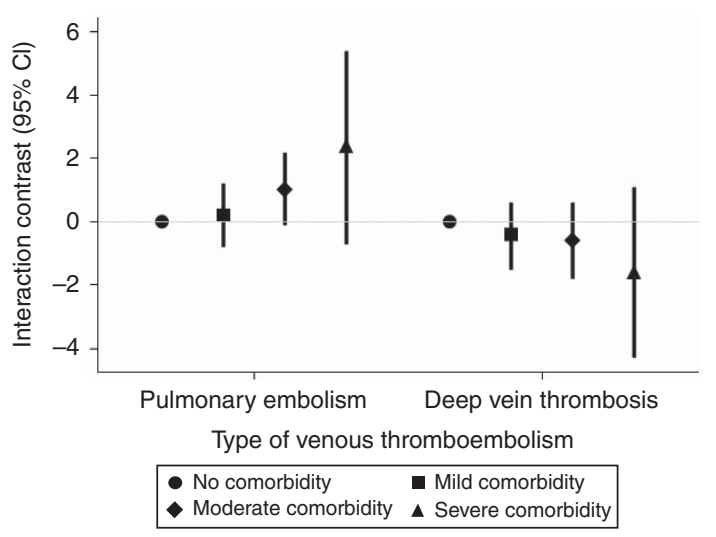

Figure 1. Interaction between CRC diagnosis and comorbidity level with respect to incidence of specific types of venous thromboembolism. Interaction contrasts and $95 \%$ confidence intervals calculated with reference to subjects in the matched comparison cohort with no comorbidity, Denmark, 1995-2010.

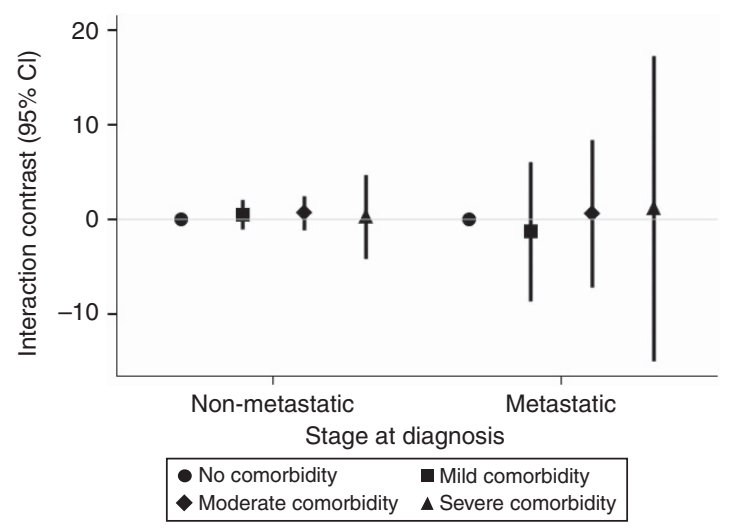

Figure 2. Interaction between CRC diagnosis and comorbidity level with respect to VTE incidence, stratified by cancer stage at diagnosis. Interaction contrasts and 95\% confidence intervals calculated with reference to subjects in the matched comparison cohort with no comorbidity, Denmark, 1995-2010. metastatic or metastatic CRC with respect to overall VTE incidence. There was no evidence of interaction between comorbidity and either CRC stage.

Of the 56189 subjects comprising the CRC cohort, 37275 (66\%) had been diagnosed with colon cancer and 18826 (34\%) had been diagnosed with rectal cancer. The remaining $88(0.2 \%)$ had been diagnosed with coincident colon and rectal cancer and were excluded from the site-specific analyses summarised in Figure 3. Colon cancer did not appear to interact with comorbidity with respect to VTE incidence. Rectal cancer appeared to interact with moderate comorbidity to increase the rate of VTE beyond the rate expected from both exposures acting independently. However, this interaction was measured with high variance and was not evident at any other level of comorbidity, and likely represents a spurious phenomenon. Inclusion of VTE cases diagnosed in the outpatient setting resulted in 119 and 312 more cases in the CRC and reference cohorts, respectively. Incidence rates and ICs from this sensitivity analysis, and from analyses within the strata of calendar period, did not differ substantially from those in the main analysis (data not shown).

\section{DISCUSSION}

We observed positive, independent associations between CRC, prevalent comorbidity level, and incidence of VTE within 5 years of CRC diagnosis. The VTE rate observed among CRC patients was on par with rates observed in a US study population (approximately 12 events per $1000 \mathrm{PY}$ at risk; Alcalay et al, 2006; Chew et al, 2006). To our knowledge, ours is the first study to include comorbidity burden and VTE incidence in a general (nonclinical) population. We noted a monotonic increase in age- and sex-adjusted VTE incidence rates, from 2.3 events per 1000 PY among individuals without comorbidity to 5.5 events per 1000 PY among individuals with severe comorbidity. The rate we observed among the general population without comorbidity is somewhat higher than the annual rate of 1 event per 1000 adults cited in the literature (White, 2003). This is likely a function of the older age distribution in our reference cohort - a consequence of matching reference subjects to CRC patients on their age at diagnosis.

We saw no evidence for biological interaction between CRC and comorbidity with respect to VTE incidence overall. However, we saw some evidence for positive interaction between CRC and comorbidity with respect to incidence of $\mathrm{PE}$, and for negative interaction between CRC and comorbidity with respect to

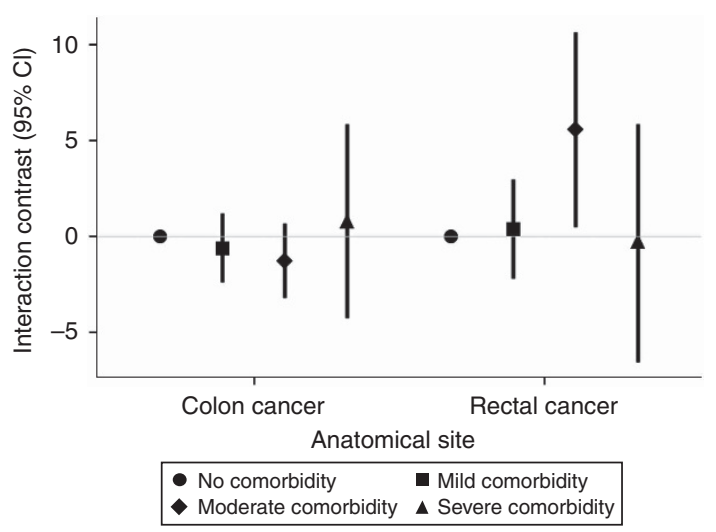

Figure 3. Interaction between CRC diagnosis and comorbidity level with respect to VTE incidence, according to anatomical site of cancer. Interaction contrasts and 95\% confidence intervals calculated with reference to subjects in the matched comparison cohort with no comorbidity, Denmark, 1995-2010. 
incidence of DVT. It should be noted that these results are based on subgroups of our main analysis, and the interaction contrasts were measured with relatively high variance; these patterns should therefore be interpreted conservatively.

Among our study's strengths are its large size and accrual of subjects from an unselected source population, which together minimised random error and the likelihood of selection bias. In addition, exposure and outcome data were ascertained from the independently maintained, nationwide, population-based medical and civil registries of Denmark. Reporting cases of malignancy to the DCR has been mandatory since 1987; validity of recorded cases is high and no false positives are expected (Gjerstorff, 2011).

Among our study's limitations are potential misclassification of comorbidity. Comorbidities were ascertained from the DNPR, which captured diagnoses made in non-psychiatric hospitals since 1977 and diagnoses made at hospital outpatient clinics and emergency departments since 1995. Therefore, patients and reference subjects whose index dates were early in our study period may have had mild comorbidities that were diagnosed at outpatient or emergency clinic visits before 1995 and thus were not captured by the DNPR. This may have attenuated contrasts made between the 'no comorbidity' category and higher categories of comorbidity. However, it is unlikely that the potentially missed diagnoses were for conditions severe enough to modify VTE risk.

We lacked data on anticoagulation therapy prescribed to members of the CRC and reference cohorts. Such data would have been useful in validating VTE diagnosis in our cohort. However, our focus on DVT and PE likely resulted in a highly specific outcome definition, limiting the number of false-positive cases. Furthermore, VTE diagnoses in the DNPR were validated against medical records in a sample of 232 prostate cancer patients, showing excellent classification parameters (sensitivity $=98.0 \%$, 95\% CI: 93.8, 99.6; specificity $=87.8 \%, 95 \%$ CI: 81.4, 92.6) (Drljevic et al, 2014). It is therefore unlikely that outcome misclassification influenced our associations. Another limitation is that we had no data on inherited and acquired coagulopathies (e.g., factor V Leiden mutation), which modify VTE risk (Kyriazi and Theodoulou, 2013). Although such conditions are likely too rare to have influenced our results, they remain a potential source of residual confounding.

Finally, we lacked data on specific chemotherapy agents used to treat CRC patients. There is evidence that both standard chemotherapy and less common adjuvant therapies (e.g., antiangiogenic drugs) modify VTE risk (Khorana and Connolly, 2009). However, it is likely that the choice and duration of these therapies mediate any interaction between comorbidity and CRC status with respect to VTE incidence. Thus adjustment for chemotherapy would be inappropriate. At the same time, part of the chemotherapy/VTE association may be owing to diagnostic bias, as repeated CT imaging may increase the sensitivity of VTE classification in patients undergoing chemotherapy.

In conclusion, CRC and comorbidity independently increase the rate of VTE. It is unlikely that these two exposures synergise or antagonise one another in dually exposed individuals. In other words, our results indicate that the clinical evaluation of VTE risk among patients with both CRC and comorbidity can be informed by the additive effects of the baseline rate in the population (those without $\mathrm{CRC}$ and without comorbidity), by the rate increase associated with prevalent CRC, and by the rate increase associated with comorbidity level.

\section{ACKNOWLEDGEMENTS}

The study was supported by a grant from the Danish Cancer Society (R73-A4284-13-S17), the Program for Clinical Research
Infrastructure (PROCRIN) established by the Lundbeck Foundation and the Novo Nordisk Foundation, and by the Danish Agency for Science, Technology and Innovation (10-084581). Thomas Ahern was supported by a Career Catalyst Award from Susan G. Komen for the Cure (CCR13264024).

\section{CONFLICT OF INTEREST}

The authors declare no conflict of interest.

\section{REFERENCES}

Alcalay A, Wun T, Khatri V, Chew HK, Harvey D, Zhou H, White RH (2006) Venous thromboembolism in patients with colorectal cancer: incidence and effect on survival. J Clin Oncol 24(7): $1112-1118$.

Andersen TF, Madsen M, Jorgensen J, Mellemkjoer L, Olsen JH (1999) The Danish National Hospital Register. A valuable source of data for modern health sciences. Dan Med Bull 46(3): 263-268.

Ay C, Dunkler D, Marosi C, Chiriac AL, Vormittag R, Simanek R, Quehenberger P, Zielinski C, Pabinger I (2010) Prediction of venous thromboembolism in cancer patients. Blood 116(24): 5377-5382.

Blom JW, Doggen CJ, Osanto S, Rosendaal FR (2005) Malignancies, prothrombotic mutations, and the risk of venous thrombosis. JAMA 293(6): 715-722.

Charlson M, Pompei P, Ales K, MacKenzie C (1987) A new method of classifying prognostic comorbidity in longitudinal studies: development and validation. J Chronic Dis 40(5): 373-383.

Chew HK, Wun T, Harvey D, Zhou H, White RH (2006) Incidence of venous thromboembolism and its effect on survival among patients with common cancers. Arch Intern Med 166(4): 458-464.

Cushman M (2007) Epidemiology and risk factors for venous thrombosis. Semin Hematol 44(2): 62-69.

Drljevic A, Borre M, Hoyer M, Ehrenstein V, Nguyen-Nielsen M (2014) Quality of venous thromboembolism diagnoses among prostate cancer patients in the Danish National Registry of Patients. Clin Epidemiol 6: 351-357.

Frank L (2000) Epidemiology. When an entire country is a cohort. Science 287(5462): 2398-2399.

Gjerstorff ML (2011) The Danish Cancer Registry. Scand J Public Health 39(7 Suppl): 42-45.

Gouin-Thibault I, Achkar A, Samama MM (2001) The thrombophilic state in cancer patients. Acta Haematol 106(1-2): 33-42.

Greenland S, Lash TL, Rothman KJ (2008) Concepts of Interaction. In Modern Epidemiology, Rothman KJ, Greenland S, Lash TL (eds) 3rd edn, Chapter 5, pp 71-83. Wolters Kluwer | Lippincott Williams \& Wilkins: Philadelphia, PA, USA.

Kahn SR, Hirsch A, Shrier I (2002) Effect of postthrombotic syndrome on health-related quality of life after deep venous thrombosis. Arch Intern Med 162(10): 1144-1148.

Khorana AA, Connolly GC (2009) Assessing risk of venous thromboembolism in the patient with cancer. J Clin Oncol 27(29): 4839-4847.

Khorana AA, Francis CW, Culakova E, Kuderer NM, Lyman GH (2007) Frequency, risk factors, and trends for venous thromboembolism among hospitalized cancer patients. Cancer 110(10): 2339-2346.

Konigsbrugge O, Pabinger I, Ay C (2014) Risk factors for venous thromboembolism in cancer: novel findings from the Vienna Cancer and Thrombosis Study (CATS). Thromb Res 133(Suppl 2): S39-S43.

Kyriazi V, Theodoulou E (2013) Assessing the risk and prognosis of thrombotic complications in cancer patients. Arch Pathol Lab Med 137(9): $1286-1295$.

Lemmens VE, Janssen-Heijnen ML, Houterman S, Verheij KD, Martijn H, Poll-Franse L, Coebergh JW (2007) Which comorbid conditions predict complications after surgery for colorectal cancer? World J Surg 31(1): 192-199.

Mukherjee D, Lidor AO, Chu KM, Gearhart SL, Haut ER, Chang DC (2008) Postoperative venous thromboembolism rates vary significantly after different types of major abdominal operations. J Gastrointest Surg 12(11): 2015-2022. 
Pedersen C, Gøtzsche H, Møller J, Mortensen P (2006) The Danish Civil Registration System. A cohort of eight million persons. Dan Med Bull 53(4): 441-449.

Prandoni P, Falanga A, Piccioli A (2005) Cancer and venous thromboembolism. Lancet Oncol 6(6): 401-410.

Schmidt M, Pedersen L, Sorensen HT (2014) The Danish Civil Registration System as a tool in epidemiology. Eur J Epidemiol 29(8): 541-549.

Sogaard KK, Schmidt M, Pedersen L, Horvath-Puho E, Sorensen HT (2014) 30 -year mortality after venous thromboembolism: a population-based cohort study. Circulation 130(10): 829-836.

Storm HH, Michelsen EV, Clemmensen IH, Pihl J (1997) The Danish Cancer Registry - history, content, quality and use. Dan Med Bull 44(5): 535-539.
White RH (2003) The epidemiology of venous thromboembolism. Circulation 107(23 Suppl 1): I4-I8.

Wun T, White RH (2009) Venous thromboembolism (VTE) in patients with cancer: epidemiology and risk factors. Cancer Invest 27(Suppl 1): 63-74.

This work is published under the standard license to publish agreement. After 12 months the work will become freely available and the license terms will switch to a Creative Commons AttributionNonCommercial-Share Alike 4.0 Unported License.

Supplementary Information accompanies this paper on British Journal of Cancer website (http://www.nature.com/bjc) 